DFPD 99/TH/31

UNICAL-TH 2/99

BITP-99-3E

July 1999

\title{
TRIPLE POMERON AND PROTON DIFFRACTION DISSOCIATION •
}

\author{
R. Fiore $^{a \dagger}$, A. Flachi ${ }^{b \ddagger}$, L.L. Jenkovszky ${ }^{c \S}$, F. Paccanoni ${ }^{d *}$, A. Papa ${ }^{a \dagger}$ \\ ${ }^{a}$ Dipartimento di Fisica, Università della Calabria, \\ Istituto Nazionale di Fisica Nucleare, Gruppo collegato di Cosenza \\ Arcavacata di Rende, I-87036 Cosenza, Italy \\ ${ }^{b}$ Physics Department, University of Newcastle upon Tyne, \\ Newcastle upon Tyne, NE1 7RU, United Kingdom \\ ${ }^{c}$ Bogoliubov Institute for Theoretical Physics, \\ Academy of Sciences of the Ukrain \\ 252143 Kiev, Ukrain \\ ${ }^{d}$ Dipartimento di Fisica, Università di Padova, \\ Istituto Nazionale di Fisica Nucleare, Sezione di Padova \\ via F. Marzolo 8, I-35131 Padova, Italy
}

\begin{abstract}
We consider proton diffraction dissociation in the dipole Pomeron model, where the Pomeron is represented by a double pole in the $J$-plane, and show that unitarity can be satisfied without decoupling of the triple Pomeron vertex. Differential and total diffractive cross sections for the reaction $\bar{p}+p \rightarrow$ $\bar{p}+X$ are analyzed and reproduced in this model.
\end{abstract}

PACS numbers: 12.40.Nn, 13.85.Ni.

${ }^{*}$ Work supported by the Ministero italiano dell'Università e della Ricerca Scientifica e Tecnologica and by the INTAS.

$\dagger$ e-mail address: FIORE,PAPA @CS.INFN.IT

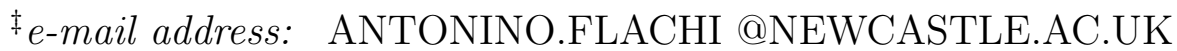

$\S$ e-mail address: JENK @BITP.KIEV.UA

*e-mail address: PACCANONI @PD.INFN.IT 


\section{Introduction}

Diffractive high energy elastic scattering for hadrons appears to find a satisfactory explanation in the framework of the Regge theory with the exchange of the Pomeron trajectory. Rising cross sections can be accounted for by assuming a Pomeron intercept slightly higher than one [1] or, in a QCD approach, by considering the Pomeron as a gluon ladder [2]. The growth of total cross sections can also be described in a way compatible with the Froissart bound in the eikonal model [3] or by assuming that the Pomeron is represented by a double pole in the complex $J$-plane 田.

In contrast with the above picture, inclusive diffractive collisions in protonproton or antiproton-proton scattering, where one of the initial particles changes only slightly its momentum and appears in the final state isolated in rapidity, seem to require deep modifications to the standard Regge models. The basic problem with diffraction dissociation, known for long [0], is that the integrated cross section $\sigma_{S D}$ appears to grow faster than the total cross section $\sigma_{T}$, thus violating unitarity. For example, in the case of a supercritical Pomeron with $\alpha(0)=1+\delta, \sigma_{S D}$ grows twice as fast, $\sim s^{2 \delta}$, as the total cross section does, $\sigma_{T} \sim s^{\delta}$. The only way to resolve this discrepancy seemed to require the vanishing of the triple Pomeron coupling (Pomeron decoupling [5]), which however contradicts the experimental data [6].

A number of different unitarization recipes have been proposed in order to modify the energy dependence of the predicted cross section. Eikonal corrections [7] succeed in reproducing the main features of single diffraction at high energy, while the same effect can be reached by the inclusion of cuts in the Regge theory [8]. Recently a different, more phenomenological, approach has been considered [9, 10, 11]. Renormalization [9, [1] or damping [11] of the Pomeron flux, that consists in setting a limit to the probability that the proton emits a Pomeron, allow for a rising of 
the total diffraction cross section compatible with experimental data. All the above approaches are based on a supercritical Pomeron input with a Pomeron intercept larger than one.

Apart from the incompatibility with the experimentally rising cross sections, a unity intercept Pomeron would present analogous problems with unitarity [5]. If, however, the partial wave amplitude, for the Pomeron exchange, presents a simple and a double pole in the complex $J$-plane, cross sections will grow with energy and it will be possible to satisfy unitarity at the Born level, without eikonalization [4]. In all (or most) of the models explored until now, the Pomeron was assumed to be an isolated single Regge pole. From QCD we know however [2] that the Pomeron is not a single pole, but rather a complicated set of singularities in the $J$-plane. A simple and feasible way to approximate this complicated structure is to take the sum of a simple and a double pole (dipole). The dipole Pomeron is known [4] to have unique properties since it reproduces itself under unitarization and thus one expects that it can be used also to resolve the abovementioned problem in diffraction dissociation. Obviously, the sum of a simple and double pole - like any combination of Regge singularities - looses factorizability, although each term remains factorizable. Since Regge pole factorization appears to be in conflict with experimental results [9], the approach we consider is favoured.

The dipole Pomeron model has been tested successfully in elastic hadron-hadron and $\gamma$-hadron reactions $4,12,13,14,15]$ and an application to single diffractive dissociation has been considered in Refs. [16, 17]. It turns out that, in this approach, the Pomeron contribution consists of two terms, one increasing like the logarithm of the energy and the other being energy independent, multiplied by "a priori" 
different $t$-dependent vertex functions. This feature, and the assumption that the Pomeron couples in a different way to Pomerons and hadrons, opens the way to a unified treatment of elastic and production amplitudes.

Since the inclusive process of hadron diffraction has been discussed extensively in the literature [5, 18, 19, 20, 21, 6] we will start, in Section 2, from the Mueller discontinuity formula and adapt it to the chosen model. The triple Pomeron contribution will be discussed in detail and the possibility to satisfy the unitarity constraint will be investigated. While a proof of the proposed solution cannot be given in the framework of the Regge theory because the $t$-dependence of the vertices is arbitrary to a great extent, plausibility arguments can be advanced on the basis of dynamical models for the Pomeron. Section 3 will be devoted to the inclusion of secondary Regge trajectories and the final expression for the cross section will be compared with experimental data in Section 4. The conclusion of this work will be drawn in Section 5.

\section{The triple Pomeron in diffractive dissociation}

Consider first the process $a+b \rightarrow c+X$ with the exchange of Regge trajectories $\{i\}$. From the Mueller discontinuity formula [22] we get

$$
\pi E_{c} \frac{d^{3} \sigma}{d \vec{p}_{c}}=\frac{1}{16 \pi s} \sum_{X}\left|\sum_{i} \beta_{a \bar{c}}^{i}(t) \xi_{i}(t) F^{i b \rightarrow X}\left(M^{2}, t\right)\left(\frac{s}{M^{2}}\right)^{\alpha_{i}(t)}\right|^{2}
$$

in the usual Regge pole model. $M^{2}$ is the squared mass of the unrevealed state $X$, $\alpha_{i}(t)$ represents the Regge trajectory exchanged and

$$
\xi_{i}(t)=\frac{1 \pm \exp \left(-i \pi \alpha_{i}(t)\right)}{\sin \left(\pi \alpha_{i}(t)\right)}
$$


is its signature. In the following $i=P, f, \pi$ and $\omega$, where $P$ stands for the Pomeron trajectory.

Consider now the elastic scattering and suppose that, asymptotically, the absorptive part in the $s$-channel, $A(s, t)$, goes like

$$
A(s, t) \propto \beta_{1}(t) \beta_{2}(t) s^{\alpha(t)}[h(t) \ln s+C],
$$

then the partial wave amplitude presents a simple and double pole in the complex $J$-plane. The amplitude for the Pomeron exchange can then be written as

$$
T(s, t) \propto-\frac{(-i s)^{\alpha(t)}}{\sin (\pi \alpha(t) / 2)} \beta_{1}(t) \beta_{2}(t)\left[h(t)\left(\ln s-i \frac{\pi}{2}\right)+C\right],
$$

where constant terms have been collected in $C$. The explicit form of $h(t)$ depends on the model. As an example, in a dual model, if the residue of the simple pole has the form $\beta(\alpha(t))$, the residue of the double pole will be given by $\int \beta(\alpha) d \alpha+$ const [4]. The form of this residue is such that the coefficient of the double pole can vanish for $t=0$, if this is required from general principles.

To substantiate this possibility, we can generalize the picture of Ref. [23] and suppose that the Pomeron pole couples to quarks through the exchange of two gluons. In order to describe the gluon-Pomeron-gluon vertex we use, at high energy, the rules of covariant Reggeization [24, 25] for the coupling of the Pomeron pole to massive vector mesons, since the gluons are off-shell. This require the introduction of five unknown functions of $t$ (some of them will vanish because of gauge invariance) that will appear in the amplitude multiplied by polynomials in $\alpha(t)$ and by appropriate powers of $\nu, \nu^{\alpha(t)-n}$, where $\nu \approx s / 2$ at high energy and $n=0,1, \ldots$. The final expression is complicated, but it can be easily seen that there will appear, among the leading contributions $\nu^{\alpha(t)}$, at least one term vanishing when $\alpha(t)=1$. 
This property remains true when we integrate over the gluon and quark momenta and, by taking the derivative with respect to $\alpha(t)$, we introduce a double pole for the Pomeron. Moreover this result does not depend on the choice of the partonic wave function of the hadron. The conclusion is that, also in elastic hadron-hadron scattering, the presence of a term vanishing with $t$, together with other terms finite at $t=0$, is highly probable in the conventional Regge residue. It could well happen that, if we consider the triple Pomeron vertex, the unitarity condition will impose constraints on the couplings such that $h(t)$ in Eq. (2) vanishes at $t=0$.

In the dipole Pomeron approach, Eq. (1) becomes

$$
\begin{aligned}
& \frac{d^{2} \sigma}{d M^{2} d t}= \\
& \frac{1}{16 \pi s^{2}} \sum_{X} \mid \beta_{a \bar{c}}^{P}(t)\left(-i \frac{s}{M^{2}}\right)^{\alpha_{P}(t)}\left[h(t)\left(\ln \frac{s}{M^{2}}-i \frac{\pi}{2}\right)+C\right] F^{P b \rightarrow X}\left(M^{2}, t\right) \\
& +\left.\sum_{i \neq P} \beta_{a \bar{c}}^{i} \xi_{i}(t) F^{i b \rightarrow X}\left(M^{2}, t\right)\left(\frac{s}{M^{2}}\right)^{\alpha_{i}(t)}\right|^{2} .
\end{aligned}
$$

Let us consider now the triple Pomeron contribution to Eq. (3), neglecting for the moment all the interference terms and replacing the sum over intermediate states by a discontinuity in $M^{2}$,

$$
\begin{aligned}
& \frac{1}{16 \pi s^{2}}\left[\beta_{a \bar{c}}^{P}(t)\right]^{2}\left(\frac{s}{M^{2}}\right)^{2 \alpha_{P}(t)} \times \\
& {\left[\left(h(t) \ln \frac{s}{M^{2}}+C\right)^{2}+\frac{\pi^{2}}{4} h^{2}(t)\right] \operatorname{Im} T^{P b}\left(M^{2}, t, \alpha_{P}(t), t_{b \bar{b}}=0\right),}
\end{aligned}
$$

where, according to Eq. (2),

$$
\operatorname{Im} T^{P b}=\sigma_{0}\left(M^{2}\right)^{\alpha_{P}(0)}\left(\lambda+\bar{h}(0) \ln M^{2}+\lambda^{\prime}\left(M^{2}\right)^{\alpha_{f}(0)-1}\right) g(t),
$$

$g(t)$ being the triple Pomeron coupling. A term, decreasing with $M^{2}$, is present in Eq. (5) since we consider also the secondary $f$ trajectory in $P-b$ scattering. 
Obviously, if $h(0)$ vanishes, the same will be true for $\bar{h}(0)$. In the following, $\alpha_{P}(t)=$ $1+\alpha^{\prime} t, \alpha^{\prime}=0.25 \mathrm{GeV}^{-2}$ and the standard form for the residue will be assumed: $\beta_{a \bar{c}}^{P}=\exp (b t)$.

By integrating Eq. (5) over $t$ and $M^{2}$ we get the Pomeron contribution to the single diffractive cross-section, $\sigma_{S D}$. We will now show that the constraint $\sigma_{S D}<\sigma_{T}$ for all values of $s$ requires that $h(t) \propto(-t)^{\gamma}$ with $\gamma>1 / 2$. Without changing the asymptotic behaviour of the Pomeron-hadron vertex, we can assume that [

$$
h(t) \propto\left(\frac{-t}{-t+1}\right)^{\gamma}, \quad \gamma \geq 0 .
$$

The proportionality constant in the expression for $h(t)$ is unessential since it can be factorized out in (田) by properly rescaling the constant $C$.

The proof becomes simpler if, according to experimental findings [9, [6], we consider the triple Pomeron vertex $g(t)$ as constant and neglect $\operatorname{Im} T^{P b}$ in Eq. (4). Then, setting $B=2\left(b+\alpha^{\prime} \ln \left(s / M^{2}\right)\right)$ and $y=\ln \left(s / M^{2}\right)$, the $t$ integral can be easily evaluated and reads

$$
\begin{gathered}
\int_{0}^{\infty} d t e^{-B t}\left[\left(\left(\frac{t}{t+1}\right)^{\gamma}+C\right)^{2}+\frac{\pi^{2}}{4}\left(\frac{t}{t+1}\right)^{2 \gamma}\right]= \\
\Gamma(2 \gamma+1) \Psi(2 \gamma+1,2 ; B)\left(y^{2}+\frac{\pi^{2}}{4}\right)+2 y C \Gamma(\gamma+1) \Psi(\gamma+1,2 ; B)+\frac{C^{2}}{B},
\end{gathered}
$$

where $\Psi(a, c ; x)$ is a confluent hypergeometric function [26].

In order to integrate over $M^{2}$ we transform to the variable $B$ whose upper limit is, asymptotically, proportional to $\ln s$. The integral can be evaluated exactly by using the elementary relations for the $\Psi$ function and, in the limit $B \sim \ln s \rightarrow \infty$,

\footnotetext{
${ }^{1}$ Other choices are possible as well: the function $h(t) \propto 1-\exp (\bar{\gamma} t)$ has a similar behaviour for suitable values of $\bar{\gamma}$.
} 
the behaviour of $\sigma_{S D}$ can be inferred from the large variable estimate for $\Psi$ [26]

$$
\sigma_{S D} \sim \Gamma(2 \gamma+1) \frac{2}{1-\gamma} B^{2-2 \gamma}+\ldots+C^{2} \ln B,
$$

where dots in the l.h.s. stand for terms with a less singular behaviour when $s \rightarrow \infty$.

We note that the singularity for $\gamma=1$ in Eq. (8) is spurious; the exact result does not present singularities for $\gamma \geq 0$. Since, in the model considered, $\sigma_{T} \sim \ln s$

and $\sigma_{S D}<\sigma_{T}$, from the first term in Eq. (8) we must have $2-2 \gamma \leq 1$. Hence, the parameter $\gamma$, in general, must satisfy the condition $\gamma \geq 1 / 2$. This inequality is necessary to avoid terms, violating unitarity, that rise faster than $\ln s$. It is important to notice that the triple Pomeron contribution does not vanish at $t=0$ because of the presence of the constant $C$.

\section{Non-leading contributions and the differential cross section}

From now on we select the hadrons participating the process: $a$ and $c$ are antiprotons $(\bar{p})$ and $b$ is a proton $(p)$. Later, for the evaluation of the total single diffractive cross section $\sigma_{S D}$, the process $a=c=p$ and $b=\bar{p}$ will be also taken into account.

On the basis of historical fits [18, 27], the $\omega$ trajectory can be neglected and, since the $\pi$ trajectory contributes in a different kinematical region with respect to $P$ and $f$, interference terms between $\pi$ and $P, f$ are suppressed. Hence, in Eq. (3) the sum over $i$ refers only to $f$, and the $\pi$ contribution will be chosen as in [6, 27, 28, 29]

$$
\left.\frac{d^{2} \sigma}{d M^{2} d t}\right|_{\pi}=\frac{1}{4 \pi} \frac{g_{\pi p p}^{2}}{4 \pi} \frac{(-t)}{\left(t-\mu^{2}\right)^{2}}\left(\frac{s}{M^{2}}\right)^{2 \alpha_{\pi}(t)-1} G^{2}(t) \sigma_{T}^{\pi p}\left(M^{2}\right),
$$


where

$$
G(t)=\frac{2.3-\mu^{2}}{2.3-t},
$$

$g_{\pi p p}^{2} /(4 \pi)=14.6$ and $\alpha_{\pi}(t)=0.9 t$.

The $f$ contribution, and its interference with the Pomeron, must now be considered. The approximation suggested in [30, 31] is based on the assumption that the $f$ couples to hadrons in just the same way as the Pomeron. This choice avoids the proliferation of free parameters and is justified from the consideration that, while the $f$ is required by the data [7, 29, 30, 31], its contribution is small, in percentage, and can be approximated. A Pomeron-Pomeron-Reggeon term larger than $0.15 \sigma_{S D}$ is excluded by high energy data [32] and is completely ignored in a recent analysis $[9]$.

Since the model we consider for the Pomeron is different from the conventional, supercritical one, we must take care in choosing an appropriate $f$ trajectory. Fits with a double Pomeron pole [13, 14, 15] require an intercept $\alpha_{f}(0)$ higher than the value, usually adopted, about 0.55 [1]. In a recent analysis [33], however, for the degenerate $a_{2} / f$ trajectory, the result $\alpha_{+}-1=-0.31 \pm 0.05$ has been obtained by refitting all the experimental cross sections considered in [1]. For all data, with errors added in quadrature, a smaller value for $\alpha_{+}$has been obtained: $\alpha_{+}-1=-0.34 \pm 0.05$. An intermediate value, -0.32 has been used in Ref. [29]. The coincidence of $\alpha_{f}(0)-1$ with -0.32 obtained in the fit of hadronic cross sections within different models for the soft Pomeron should not be surprising; in a limited energy range a behaviour $s^{\epsilon}$, for $\epsilon$ sufficiently small, can be well approximated by a term of the form $(u+v \ln s)$. 
Let $a(t)$ be the difference between the $P$ and $f$ trajectories. If we set

$$
a(t)=\alpha_{P}(t)-\alpha_{f}(t)=a(0)-\delta t
$$

then typical values, adopted in the following, are $a(0) \simeq 0.34$ and $\delta \simeq 0.65$. The $f$ contribution

$$
\begin{aligned}
R(s, t)= & k\left\{\left[h(t) \ln \frac{s}{M^{2}}+C\right] \cos \left(\frac{\pi a(t)}{2}\right)-\right. \\
& \left.\frac{\pi h(t)}{2} \sin \left(\frac{\pi a(t)}{2}\right)\right\}\left(\frac{s}{M^{2}}\right)^{-a(t)}+k^{2}\left(\frac{s}{M^{2}}\right)^{-2 a(t)}
\end{aligned}
$$

will appear in the final form of the differential cross section:

$$
\begin{aligned}
\frac{d^{2} \sigma}{d t d M^{2}} & =\frac{A}{M^{2}} e^{2\left(b+\alpha^{\prime} \ln \left(s / M^{2}\right)\right) t}\left[\left(h(t) \ln \frac{s}{M^{2}}+C\right)^{2}\right. \\
& \left.+\frac{\pi^{2}}{4} h^{2}(t)+R(s, t)\right]\left(1+l\left(M^{2}\right)^{\alpha_{f}(0)-1}\right) \\
& +\frac{1}{4 \pi} \frac{g^{2}}{4 \pi M^{2}} \frac{(-t)}{\left(t-\mu^{2}\right)^{2}} G^{2}(t)\left(\frac{s}{M^{2}}\right)^{2 \alpha_{\pi}(t)-2} \sigma_{T}^{\pi p}\left(M^{2}\right),
\end{aligned}
$$

where all the constant factors have been collected in $A$.

In Ref. [30] a value near 7.8 is quoted for the parameter $k$, appearing in $R(s, t)$; since, however, the expression (11) has been rescaled, $k$ is here a new parameter. As far as the other parameters are concerned, $b$ will be fixed from $p-p$ elastic scattering (e.g. $b=2.25 \mathrm{GeV}^{-2}$, consistent with the slopes used in [9, 12, 13, 14, 15, [7]) and $\sigma_{T}^{\pi p}\left(M^{2}\right)$ in the dipole Pomeron model can be written as

$$
\sigma_{T}^{\pi p}\left(M^{2}\right)=0.565+2.902 \ln \left(M^{2}\right)+44.388\left(M^{2}\right)^{\alpha_{f}(0)-1},
$$

inspired by the parametrization used in [1]. Since the form of $h(t)$ is determined only near $t=0$, it is well possible that the $t$-dependence of the cross-section should be corrected. Hence, a different value of $b$ could be required from the experimental data, but this possibility will not be considered in the following. 


\section{Comparison with data}

When comparing the model with experimental data, we find two kinds of problems. The first one is related to the experimental definition of single diffraction dissociation. The great variety of phenomenological models, adopted by different experimental groups in order to extract the published data, makes the test of any new model difficult. Moreover, integrated cross-sections do not refer to the same intervals of $M^{2}$ and $t$, for different experimental analyses. The second kind of problem resides in our parametrization and is strongly related to the first one. The integrated cross section cannot be given in compact form and, since the overall normalization of the data has an experimental uncertainty of $15 \%$, it is not an easy task to determine the parameter $\gamma$ only from the $t$-dependence of the cross sections at different energies.

While the pion contribution can be fixed as in Section 3, the parameters relative to the $f$ trajectory are different with respect to those of Refs. 30, 31, since the Pomeron contribution differs from the one proposed there. We are left with three parameters for the Pomeron and one for the $f$, plus an overall constant multiplying these contributions, while the $\pi$ term has no free parameters.

From now on, we adopt the standard variable $\xi \equiv M^{2} / s$, that represents the fraction of the momentum of the proton carried by the Pomeron. Using the expression (11) for $d^{2} \sigma / d \xi d t$ in our model, we performed a global fit of the data at $\sqrt{s}=14$ and $20 \mathrm{GeV}$ of E396 [34 and at $\sqrt{s}=546$ and $1800 \mathrm{GeV}$ of the CDF collaboration [32]. All the data were taken from the compilation of Ref. [9] and are at fixed $t=-0.05 \mathrm{GeV}^{2}$. The range of $\xi$ for the data of E396 has been limited to 
$0.0160 \div 0.1013$; in the case of the CDF data, we have considered $\xi$ in the range $0.0064 \div 0.109$ for the data at $\sqrt{s}=546 \mathrm{GeV}$ and in the range $0.0033 \div 0.0918$ for those at $\sqrt{s}=1800 \mathrm{GeV}$. We have found that our proposed model nicely fits all the data for a large range of values of the parameter $\gamma$ larger than $1 / 2$. This weak dependence on the value of the parameter $\gamma$ was not unexpected, since the fit was performed at fixed $t$. In the particular case of $\gamma=2$ (which will be justified in the following) the fit gives for the remaining parameters the following values: $C=0.9802, A=1.9080, k=0.9839$ and $l=2.3987$, with $\chi^{2} /$ d.o.f. $\approx 0.9$. In Fig. 1 we compare the curve resulting from the fit with $\gamma=2$ with the experimental data. We can see that our model succeeds in reproducing the experimental data at different values of $s$. We have checked that choosing a different value for $\gamma$ produces only little changes of the other parameters, but does not affect in a sizeable way the shape of the fitting curves.

We have then fixed the parameters in the expression for $d^{2} \sigma / d \xi d t$ in our model according to the result of the fit at $t=-0.05 \mathrm{GeV}^{2}$ and have checked how it reproduces other sets of data, obtained at different $t$ values. We have considered the data of Ref. 35] at $t=-0.015 \mathrm{GeV}^{2}$ and those of the UA8 collaboration 29] at the relatively large value of $t=-0.95 \mathrm{GeV}^{2}$. In both cases our curves roughly reproduce the data (see Figs. 2 and 3), thus indicating that also the $t$-dependence in our model is quite reasonable.

Finally, we have considered the total single diffractive cross section $\sigma_{S D}$, for the process $p(\bar{p})+p \rightarrow p(\bar{p})+X$ as a function of $\sqrt{s}$. We have compared our model with the experimental data of [32, 36, 37, 38, 39] from the compilation given in [9], where some data have been corrected in order to obtain the diffraction cross section 
for $\xi \leq 0.05$. In order to make the comparison, we have numerically integrated our expression for $d^{2} \sigma / d \xi d t$, with the parameters determined by the previous fit, in the region $1.4 / s \leq \xi \leq 0.05$ and $t \leq 0$. In Fig. 4 we observe that the result of the integration, plotted as a function of $\sqrt{s}$, is in good agreement with the experimental data over all the range of values of $s$, including the Tevatron energies $\sqrt{s}=546$ and $1800 \mathrm{GeV}$. We must stress here that the choice $\gamma=2$ is essential: values of $\gamma$ lower than 2, but larger than $1 / 2$ in order to satisfy $\sigma_{S D}<\sigma_{T}$, would give a too fast growth with $s$, whereas larger values of $\gamma$ would cause an undershooting of the data at large $s$.

\section{Conclusions}

In this paper we have considered the proton diffraction dissociation in the dipole Pomeron model. In this model the differential cross section $d^{2} \sigma / d \xi d t$ can be written in the form given in Eq. (11). From the theoretical point of view, the result in Eq. (11) assesses two important properties that seem to be required by the data [9]. First, the exact factorization, typical of the Regge pole model, is lost in the dipole Pomeron approach. Second, for $t=0$ the Pomeron and pion contributions are independent of $s$ and the scaling with $M^{2}$ of $d^{2} \sigma /\left.d M^{2} d t\right|_{t=0}$ becomes exact if only these terms are considered. Moreover, we remark that this model respects the unitarity condition without decoupling of the triple Pomeron vertex. The total diffractive cross section rises as $\ln (\ln s)$, i.e. slower than the total $p-\bar{p}$ cross section that, in turn, satisfies the Froissart bound.

We notice that, in Eq. (11), the triple Pomeron coupling and the Pomeronproton cross section are tangled in the multiplicative constant $A$ together with an 
unknown scale factorized from the function $h(t)$. Hence the fit of the experimental data cannot determine the aforesaid quantities but, at any rate, it represents an important test of the model. Concerning the comparison with experimental data, we have found that this model gives a satisfactory fit to the experimental data for $d^{2} \sigma / d \xi d t$ with regards both to the $\xi$ - and $t$-dependence. Moreover, for a suitable choice of the parameter $\gamma$, it well reproduces also the data for the total single diffractive cross section and allows to predict a value of about $11 \mathrm{mb}$ at the LHC energy $\sqrt{s}=14 \mathrm{TeV}$.

We stress that in our model the one-pion contribution, parametrized in Eq. (9), has been fixed from the beginning, differently from Ref. [9], where a multiplicative constant has been considered in front of it as one of the two free parameters to be fitted. As for the $f$ contribution, in our model it is well below the limit found by $\mathrm{CDF}$ [32]. The discrepancies observed at large $\xi$ from the data of Ref. [35] and of UA8 [29] could arise from an underestimation of the contribution of the $\pi$ and from neglecting that of the $\omega$. According to Ref. [29], the one-pion exchange contribution is only a small part of the total non-Pomeron exchange background. Also the approximated treatment of the $f$ could be responsible for the disagreement at large $\xi$. What we need is a more rigorous method for justifying the $t$-dependence of our parametrization and an extensive study of non-leading contributions. We feel that a deeper insight in these problems is important for applications of the model to other processes. 


\section{Acknowledgment}

One of us (L.L.J.) is grateful to the Dipartimento di Fisica dell'Università della Calabria and to the Istituto Nazionale di Fisica Nucleare - Sezione di Padova e Gruppo Collegato di Cosenza for their warm hospitality and financial support. 


\section{References}

[1] A. Donnachie and P.V. Landshoff, Phys. Lett. B296, 227 (1992).

[2] L.N. Lipatov, Zh. Eksp. Teor. Fiz. 90, 1536 (1986) [Sov. Phys. JETP 63, 904 (1986)];

E.A. Kuraev, L.N. Lipatov and V.S. Fadin, ibidem 72, 377 (1977) [45, 199 (1977)]

[3] T. Chou and C.N. Yang, Phys. Rev. 170, 1591 (1968);

L. Durand and R. Lipes, Phys. Rev. Lett. 20, 637 (1968).

[4] A.I. Bugrij et al., Fortschr. Phys. 21, 427 (1973); Z. Phys. C4, 45 (1980);

L.L. Jenkovszky, Fortschr. Phys. 34, 791 (1986); Riv. Nuovo Cim. 10, 1 (1987).

[5] R.C. Brower and J.H. Weis, Rev. Mod. Phys. 47, 605 (1975).

[6] K. Goulianos, Phys. Rep. 101, 169 (1983).

[7] E. Gotsman, E.M. Levin and U. Maor, Phys. Rev. D49, R4321 (1994).

[8] A.B. Kaidalov, L.A. Ponomarev and K.A. Ter-Martyrosian, Sov. J. Nucl. Phys. 44, 468 (1986).

[9] K. Goulianos and J. Montanha, Phys. Rev. D59, 114017 (1999).

[10] K. Goulianos, Phys. Lett. B358, 379 (1995) (Erratum: ibidem 363, 268 $(1995))$.

[11] E. Erhan and P. Schlein, Phys. Lett. B427, 389 (1998).

[12] M. Bertini et al., Riv. Nuovo Cim. 19, 1 (1996).

[13] P. Desgrolard et al, Nuovo Cim. 107A, 637 (1994).

[14] P. Desgrolard, A.I. Lengyel and E.S. Martynov, Nuovo Cim. 110A, 251 (1997).

[15] P. Desgrolard et al, hep-ph/9811393. 
[16] L.L. Jenkovszky, E.S. Martynov and F. Paccanoni, Proceedings of "Hadrons96", Novy Svet, Crimea, ed. by G. Bugrij et al., Kiev 1996, p. 159.

[17] R. Fiore, L.L. Jenkovszky and F. Paccanoni, Heavy Ion Phys. 9, 5 (1999).

[18] D.P. Roy and R.G. Roberts, Nucl. Phys. B77, 240 (1974).

[19] P.D.B. Collins, An Introduction to Regge Theory and High-Energy Physics, Cambridge University Press, Cambridge 1977.

[20] A.B. Kaidalov, Phys. Rep. 50, 157 (1979).

[21] G. Alberi and G. Goggi, Phys. Rep. 74, 1 (1981).

[22] A.H. Mueller, Phys. Rev. D2, 2963 (1970)

[23] F.E. Low, Phys. Rev. D12, 163 (1975);

S. Nussinov, Phys. Rev. Lett. 34, 1286 (1975).

[24] F.D. Gault and H.F. Jones, Nucl. Phys. B30, 68 (1971);

F.D. Gault and M.D. Scadron, Nucl. Phys. B15, 442 (1970).

[25] P.M. Osborne and J.C. Taylor, Nucl. Phys. B10, 213 (1969).

[26] A. Erdélyi et al., Higher transcendental functions Vol. I, McGraw-Hill (1953).

[27] R.D. Field and G.C. Fox, Nucl. Phys. B80, 367 (1974).

[28] M. Bishari, Phys. Lett. 38B, 510 (1972).

[29] UA8 Coll., A. Brandt et al., Nucl. Phys. B514, 3 (1998).

[30] A. Donnachie and P.V. Landshoff, Nucl. Phys. B244, 322 (1984).

[31] A. Donnachie and P.V. Landshoff, Phys. Lett. 191B, 309 (1987); Nucl. Phys. B303, 634 (1988).

[32] CDF Coll., F. Abe et al., Phys. Rev. D50, 5535 (1994).

[33] J. Cudell, K. Kang and S.K. Kim, Phys. Lett. B395, 311 (1997).

[34] R.L. Cool et al., Phys. Rev. Lett. 47, 701 (1981). 
[35] Y. Akimov et al., Phys. Rev. Lett. 39, 1432 (1977).

[36] J. Schamberger et al, Phys. Rev. D17, 1268 (1978).

[37] M.G. Albrow et al., Nucl. Phys. B108, 1 (1976); Nucl. Phys. B54, 6 (1973); Nucl. Phys. B72, 376 (1974).

[38] J.C.M. Armitage et al., Nucl. Phys. B194, 365 (1982).

[39] D. Bernard et al, Phys. Lett. B186, 227 (1987). 

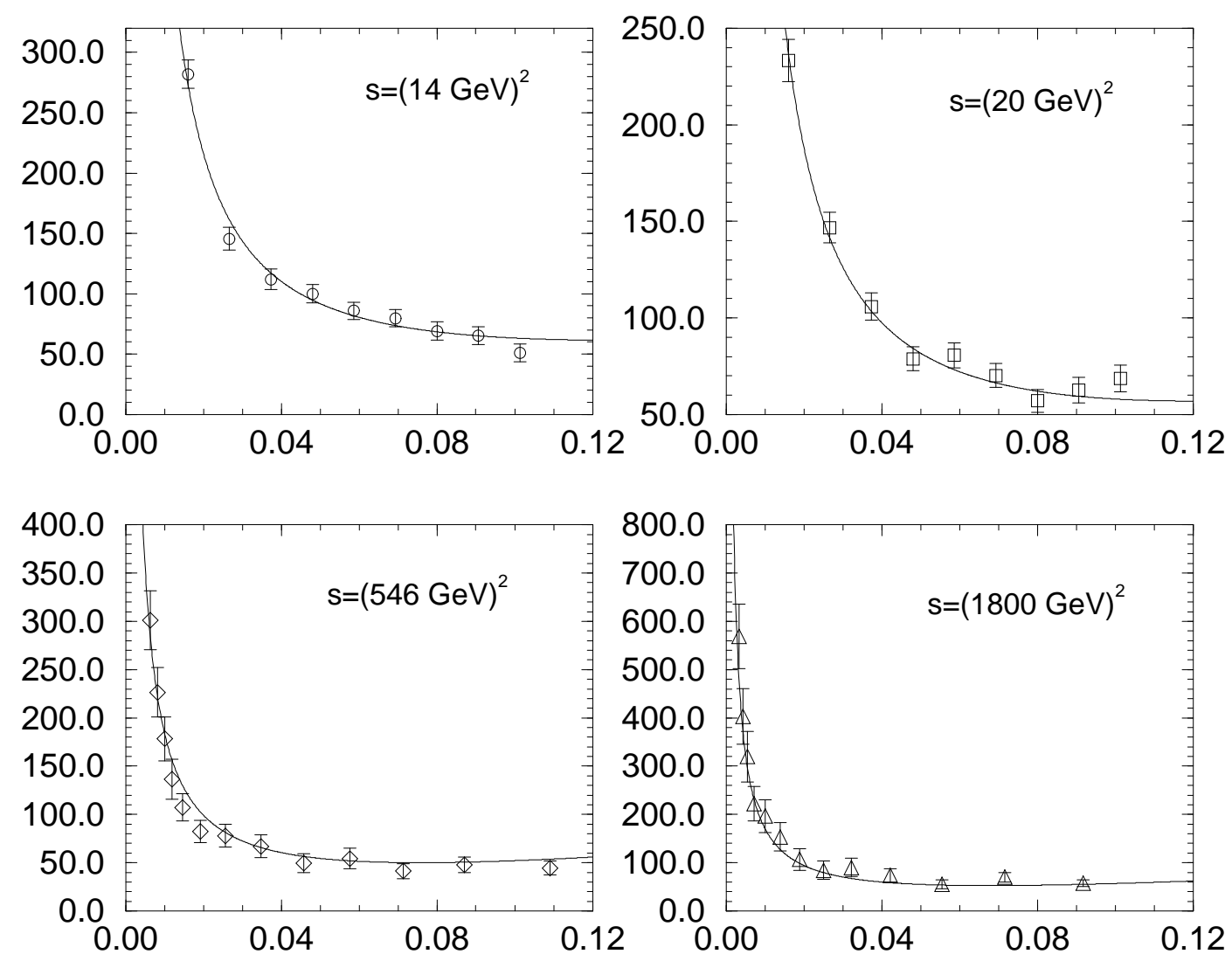

Figure 1: Differential cross sections $d^{2} \sigma / d \xi d t\left[\mathrm{mb} / \mathrm{GeV}^{2}\right]$ vs $\xi$ for several values of $s$. Data are from E396 [34] and CDF [32], compiled in [9]. The solid curves represent the model with $\gamma=2$ and with the remaining parameters determined by the fit. 

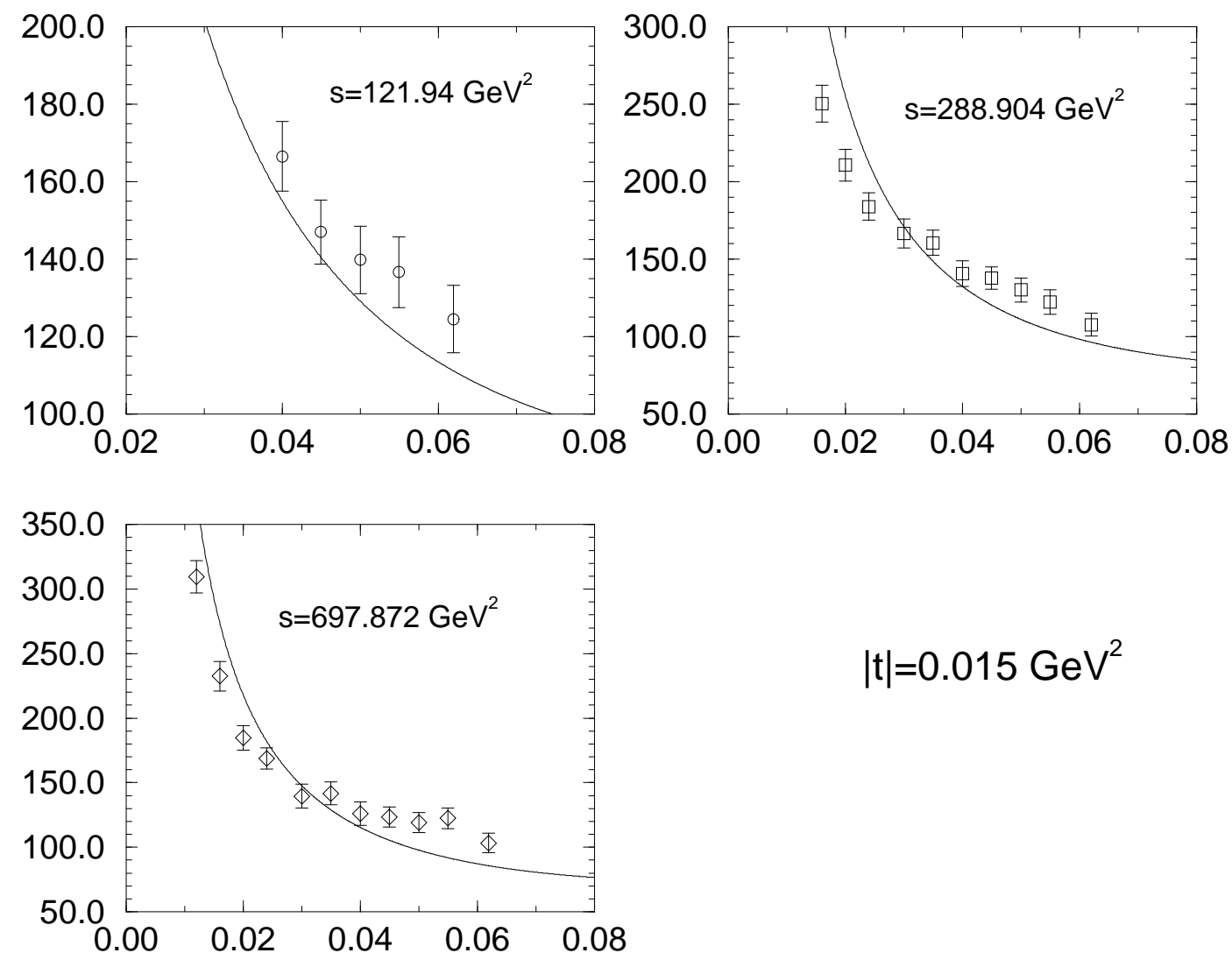

$$
|\mathrm{t}|=0.015 \mathrm{GeV}^{2}
$$

Figure 2: Differential cross sections $d^{2} \sigma / d \xi d t\left[\mathrm{mb} / \mathrm{GeV}^{2}\right]$ vs $\xi$ for several values of $s$ and $t=-0.015 \mathrm{GeV}^{2}$. The solid curves represent the model with $\gamma=2$ and with the other parameters determined by the fit shown in Fig. 1. Data are from [35] and were not included in the fit. 


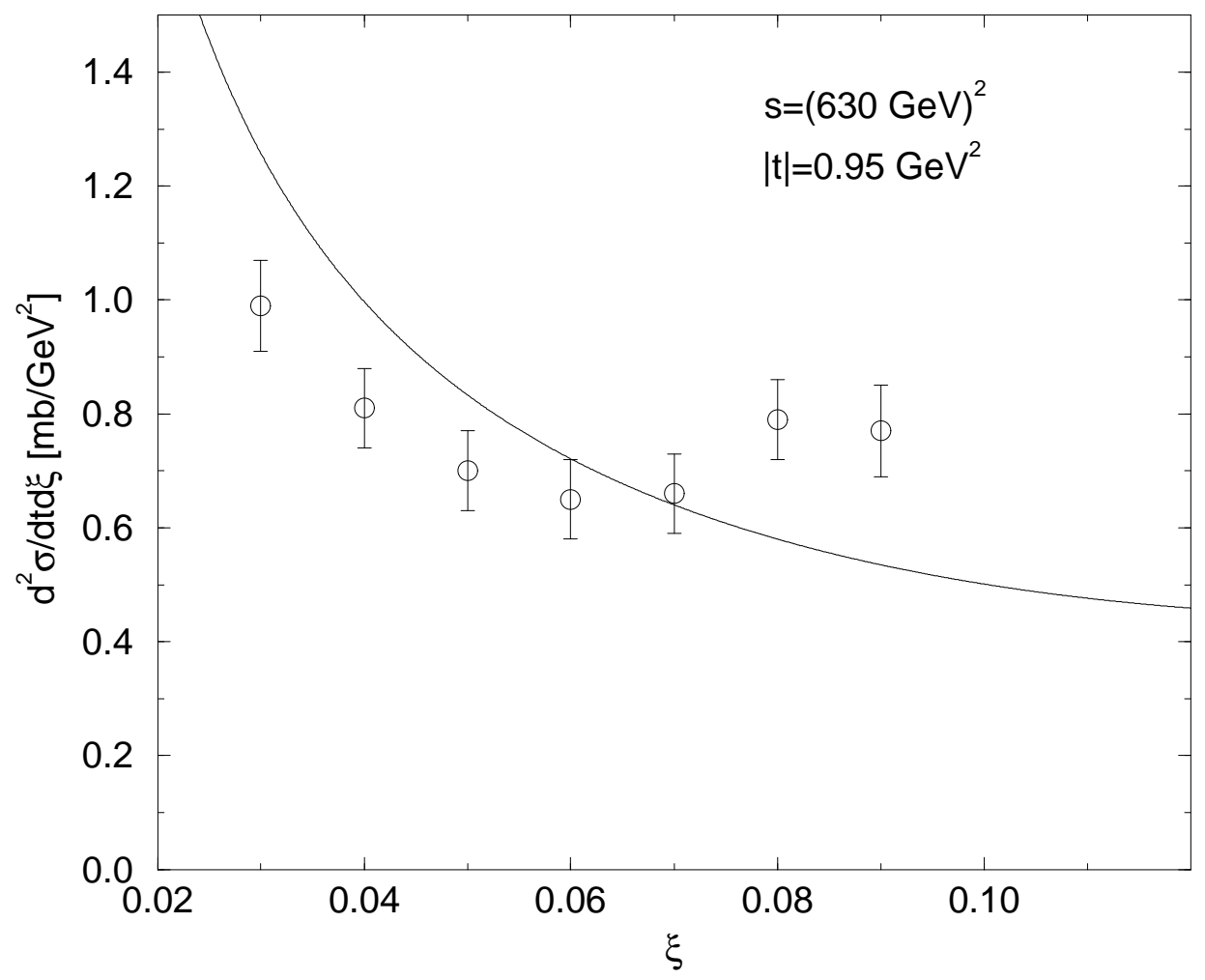

Figure 3: Differential cross sections $d^{2} \sigma / d \xi d t\left[\mathrm{mb} / \mathrm{GeV}^{2}\right]$ vs $\xi$ for $\sqrt{s}=630 \mathrm{GeV}$ and $t=-0.95 \mathrm{GeV}^{2}$. The solid curves represent the model with $\gamma=2$ and with the other parameters determined by the fit shown in Fig. 1. Data are from 29] and were not included in the fit. 


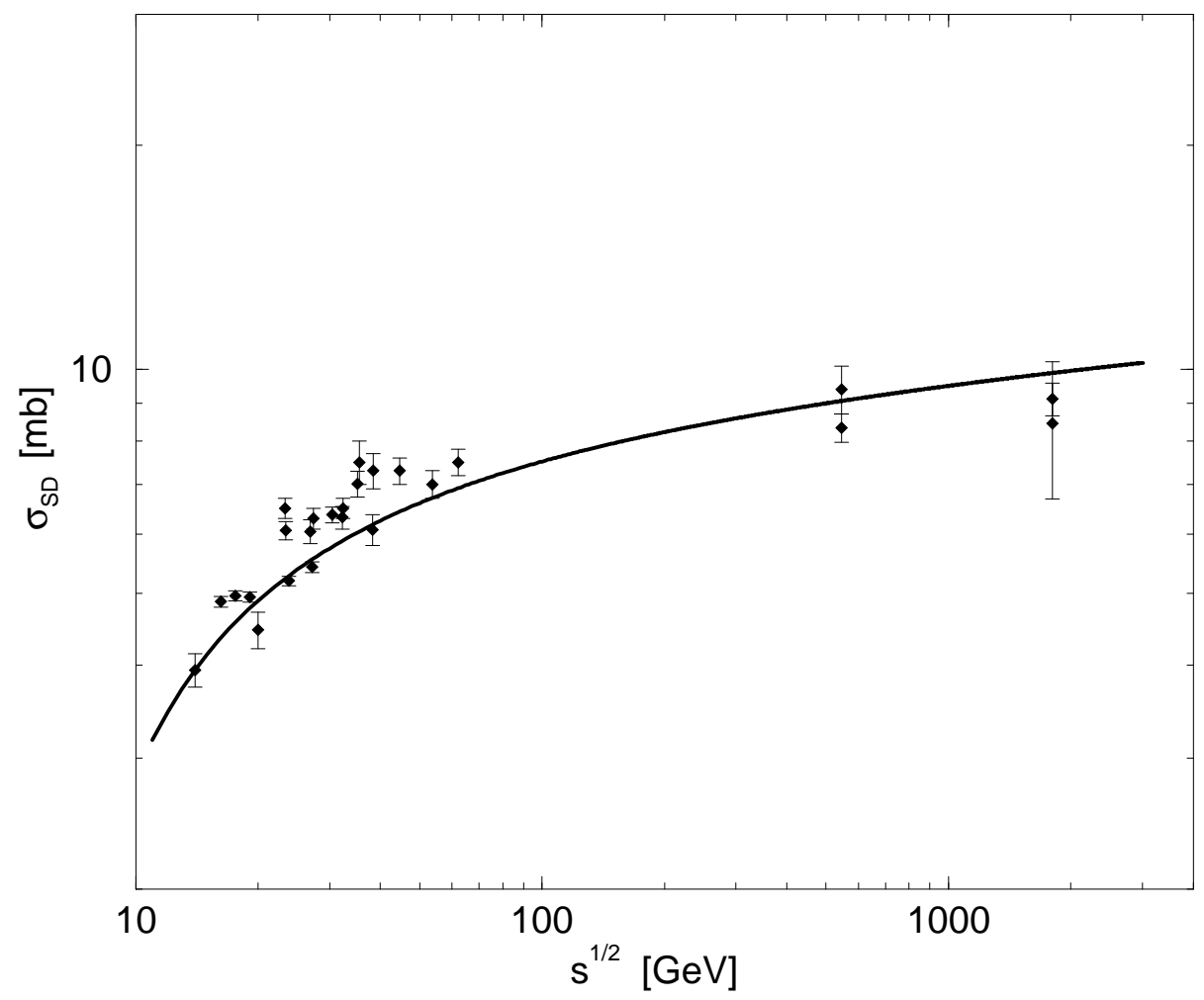

Figure 4: Total single diffraction cross section $\sigma_{S D}$ vs $\sqrt{s}$, compared with the prediction of the model. Data are taken from the compilation of Ref. [9]. 